\title{
31 Health risks from heating foods I
}

Our standard foods can be burdened with toxins from their multiple processing stages. Pesticides, aflatoxins from molds, solanine in the green spots on tomatoes, antibiotics administered to livestock or dioxins in meat and eggs are further examples of repeated food issues triggering concern by the public. Apparently, the discussions about a health-conscious diet have given less attention to the toxins that can be formed, for example, by the way we prepare our meals during the heating of certain foods.

The polycyclic aromatic hydrocarbons (PAHs) have long been an issue in this connection. One typical representative of this drug class is benzo(a) pyrene, a compound deriving from the highly toxic benzene and a potent carcinogen. Benzo(a)pyrene occurs as a product of many incomplete combustion processes and is also present, among others, in cigarette smoke ( $\triangleright$ Chapter 93 ) and in automotive exhaust fumes. When such compounds develop in smokers or particularly when grilling over charcoal that is not thoroughly glowing, they can precipitate on the grilled meat and build up. Melting fat and fat droppings on the coals facilitate the formation of PAHs. Therefore, important protection interventions like a grill with a lateral arrangement and wrapping the food to be grilled in aluminum foil should be used.

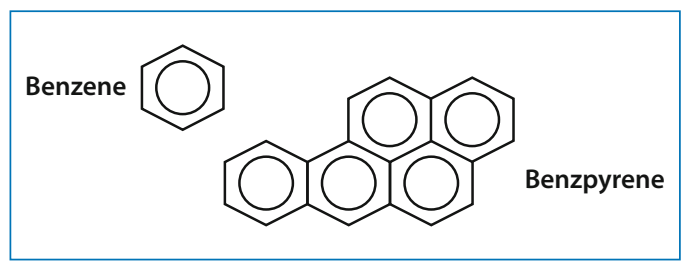

Fig. 31.1

Heterocyclic aromatic amines (HAA) increase cancer risk (Zheng et Lee 2009). They are formed from amino acids and creatine when meat from beef, pork and fowl or fish are processed for longer periods at temperatures higher than $150^{\circ} \mathrm{C}$.

Just to play it safe, darkly smoked and solid burned crusts should always be cut off.

Giving in to the desire to eat grilled meat along with darkly toasted bread or bread with very dark crust is tempting fate with additional health hazards. The is due to the monochloropropanediol (3-MCPD), a carcinogenic substance produced from table salt and from the fat cleaved from a glycerol moiety at high heat. 